\title{
IMPORTANCIA DE LAS PYMES PARA EL DESARROLLO ECONÓMICO DE CHILE EN EL SIGLO XXI
}

\author{
María Alejandra Taborda Ocampo \\ Escuela de Negocios, Gestión y Sostenibilidad \\ Programa de Especialización en Gestión Empresarial \\ Especialista en Gestión Empresarial \\ jgc@hotmail.com \\ Lady Dayana Nova \\ Escuela de Negocios, Gestión y Sostenibilidad \\ Programa de Especialización en Gestión Empresarial \\ Especialista en Gestión Empresarial \\ yayis19839@hotmail.com \\ Ingrid Viviana Bohórquez Sanabria \\ Escuela de Negocios, Gestión y Sostenibilidad \\ Programa de Especialización en Gestión Empresarial \\ Especialista en Gestión Empresarial \\ imerlina62@gmail.com
}

\section{Resumen}

El presente artículo analizará el aporte que han tenido la pymes en el desarrollo económico de Chile a partir de una investigación en las diferentes entidades que contribuyen a su desarrollo y quienes muestran sus indicadores como lo son el Centro de Negocios de Santiago la CEPAL y el SII.

Las pymes en Chile han jugando un papel fundamental para su economía ya que contribuyen en gran parte a la generación de empleos y participación en la producción nacional del país, aunque presentan una problemática para su emprendimiento en temas de financiamiento y competitividad no es un factor de restricción para su crecimiento ya que cuentan con entidades que contribuyen a su desarrollo y sostenimiento.

A continuación se observaran temas en cuanto a su contribución seguido de la problemática a la cual deben enfrentarse en temas de financiación, entidades que apoyan su desarrollo y crecimiento finalizando con su conclusión. 
Palabras clave: emprendimiento; pyme; desarrollo empresarial.

\section{Introducción}

Al analizar el entorno económico de un país, se encuentra que está compuesto de diversos sectores unos más dinámicos que otros, esto se debe, a la fortaleza de producción que presenta cada nación convirtiéndose en una ventaja competitiva respecto a otros países. Asî mismo, encontramos en dichos sectores pequeñas, medianas y grandes empresas que deben estar preparadas para replantearse y responder acertadamente a los cambios que ocurren en el mercado. Lo anterior, resulta de gran importancia porque estos cambios a su vez pueden afectar la economía de un país. Es por esto, que las pymes tienen una razón de ser a nivel mundial, ya que, pueden responder más ágilmente a tipo de cambios.

De acuerdo con lo referido, las pymes son una pieza fundamental de la vida económica y social en Chile, ya que aportan gran parte de la producción nacional y la generación de empleo. Las financiaciones de este tipo de empresas provienen del sector bancario, mercado que en ocasiones impone restricciones e impide su crecimiento debido a los parámetros exigidos a la hora del acceso a crédito. Sin embargo, dicha problemática no ha impedido el desarrollo de este sector.

De igual manera, estas pequeñas y medianas empresas desempeñan un papel importante en el proceso de cambio de un país, son fuente de innovación, productividad y tecnología, desempeñándose como agentes de cambio en una economía globalizada.

Ahora bien, el presente artículo busca analizar la importancia que tienen las pequeñas y medianas empresas en Chile, cuál ha sido el apoyo público y privado obtenido para su crecimiento y con cuales alternativas de financiación cuentan, así mismo, las principales problemáticas por las que han tenido que pasar en su dinámica empresarial contando con su proyección la cual concluye su evolución.

Lo anterior se realiza con el ánimo de determinar el impacto que generan dichas empresas en la economía de este país.

\section{Aspectos generales de la economía de Chile}

Según el banco mundial, chile ha sido de las economías de más rápido crecimiento en Latinoamérica, lo cual ha permitido una reducción considerable de la pobreza, esto debido a los avances en los programas de acceso a la educación, pasando de un índice de $38 \%$ en 1990 a 14,4\% en 2011 y $10 \%$ en el 2016, además a través de análisis a la actividad económica, la Comisión Económica para América Latina y el Caribe (CEPAL) Ubicó a chile en el segundo lugar como el país con menor índice de pobreza, indicando así que solo el $1 \%$ de la población habita en viviendas de materiales precarios. 


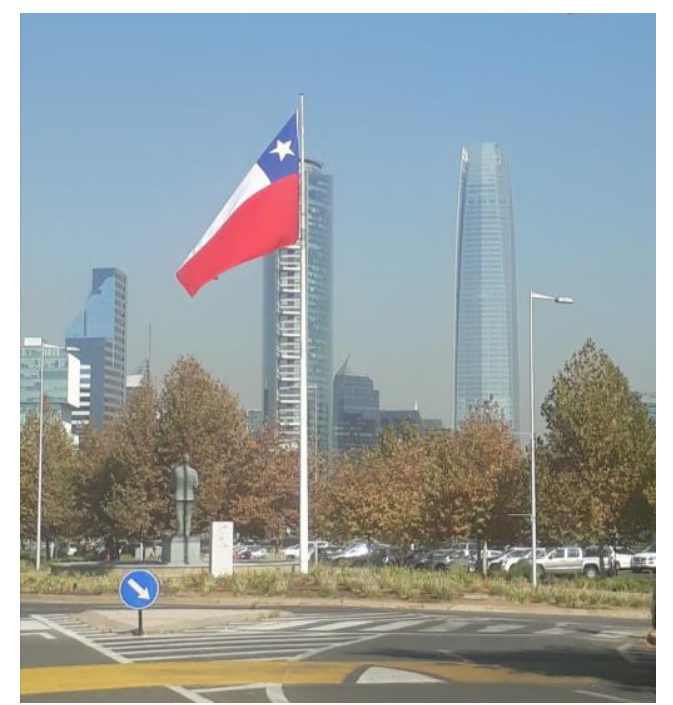

Figura 1. Comisión Económica para América Latina y el Caribe CEPAL, Santiago de Chile. Fuente: Los autores

Así mismo, el foro económico mundial afirma que chile se mantiene como líder en competitividad en américa latina y el caribe, el estudio revela que se mantiene en el trigésimo tercer puesto del ranking mundial compuesto por 137 países. (El mostrador, 2017). El portal web: This is chile, ratifica que la estabilidad es lo que mejor define su economía, ya que el mercado estimula la competencia y el libre comercio, muestra de esto es la firma de TLC con casi dos tercios de la población mundial. Sus industrias tradicionales tales como: la agropecuaria, vinícola, pesquera, minera han tomado un giro más innovador, sostenible y sustentable.

Un punto esencial dentro de la economía mundial son los negocios familiares, en chile estas aportan un $60 \%$ al PIB del país y un $60 \%$ de empleo, según un estudio realizado por Tharawat Magazine, se destaca que estas empresas muestran mayor rentabilidad a largo plazo, lo que significa que tienen menos posibilidades de despedir a las personas y más factibilidad de contratar aun en recesión económica. (El mostrador, 2017).

\begin{tabular}{l|lll} 
Tamaño & $\begin{array}{l}\text { No } \\
\text { familiar }\end{array}$ & Familiar & Total \\
\hline Micro & $51,3 \%$ & $48,7 \%$ & $100 \%$ \\
Pyme & $51,4 \%$ & $48,6 \%$ & $100 \%$ \\
Grande & $60,2 \%$ & $39,8 \%$ & $100 \%$ \\
Total & $51,7 \%$ & $48,3 \%$ & $100 \%$
\end{tabular}

Tabla 1. Empresas familiares según tamaño

Fuente: Cuarta Encuesta Longitudinal de Empresas, 2017

Sebastián Piñera, presidente de Chile, promueve dentro de su gobierno medidas que busquen beneficiar las pequeñas y medianas empresas (pymes). Dado que uno de los mayores 
problemas que sufren las pymes es que no les pagan a tiempo, la más reciente medida fue disminuir los pagos de las facturas a 60 días, esto con el fin de dar un alivio financiero de aproximadamente US\$80 millones para las pymes. (América Economía, 2018). Otra de las medidas tomadas por Piñeros a principios de abril fue normalizar el sistema de ingreso de emigrantes al país, esto debido a que consideran que tener extranjeros es un valor agregado, según cuenta realizada por AVLA una compañía de soluciones financieras para empresas y pymes el $67 \%$ de la población encuestada cree que chile da suficientes oportunidades laborales a los extranjeros. (Fajardo Cabello, 2018)

\section{Importancia de las pymes en Chile}

Años atrás la pequeña y mediana empresa no se consideraba como un factor importante en la economía del país, sin embargo, con el tiempo se empieza a ver su crecimiento nacional e internacional y su aporte económico, lo que las posiciona como un factor fundamental en su economía, haciendo que las Entidades gubernamentales pongan sus ojos en ellas, es allí cuando se crean organizaciones que promueven su crecimiento emprendedor y dichas empresas empiezan a tomar un mayor auge.

Las pymes nacen en chile por alguna oportunidad de mercado o necesidad generada por los consumidores, la pequeña y mediana empresa muestra una realidad económica por su aporte en generación de empleos, participación en la producción nacional, iniciativas de innovación y competitividad, lo que hace que se genere un crecimiento en el país.

El éxito del crecimiento de las pymes a pesar de las complicaciones y restricciones en los créditos se debe a cómo implementan la solución a los problemas, por ejemplo, implementan prácticas para asociarse entre ellas, lo que les permite compartir costos y ser más competitivas en el sector económico en el que mueven.

Las empresas en Chile el $97,7 \%$ son propiedad privada, $1,4 \%$ son de propiedad privada extranjera, $0,5 \%$ son de propiedad estatal, en tanto el 0,9\% restante se reparte en empresas de propiedad mixta (Fuente Cuarta encuesta longitudinal de empresas unidad de estudios Agosto 2017 Las Pymes constituyen el 98\% de las que existen en Chile, de acuerdo con la información estadística que maneja el Servicio de Impuestos Internos de Chile publicada en el año 2016, en el país existen 1.074.040 empresas de las cuales 1.059.868 están representadas en micro, pequeñas y medianas empresas, estas aportan más de 5 Millones de empleos directos (fuente Articulo Pyme.emol.com 17 Abril 2017), lo que genera un mayor desarrollo humano en las regiones que se concentran.

Estas empresas están distribuidas en los diversos sectores de la economía y debido a su composición ingresan al mercado con un proceso más lento que las grandes empresas, teniendo en cuenta que las Pymes que tienen éxito tardan hasta 10 años en su proceso de desarrollo, por ejemplo aquellas empresas que inician su incursión en el mercado lo hacen de forma pequeña para determinar un comportamiento inicial, algunas lo logran y obtienen resultados eficientes generando un crecimiento significativo y otras solo se mantienen en el mercado local. 
De acuerdo a la Cuarta Encuesta Longitudinal de Empresas ELE4 en Chile la composición de empresas por sector y tamaño se encuentran estructuradas en cinco sectores principales: comercio $(34,5 \%)$; transporte y almacenamiento

$(10,5 \%)$; industrias manufactureras $(10,2 \%)$, agrosilvicultura y pesca $(10,1 \%)$ y construcción $(9,8 \%)$.

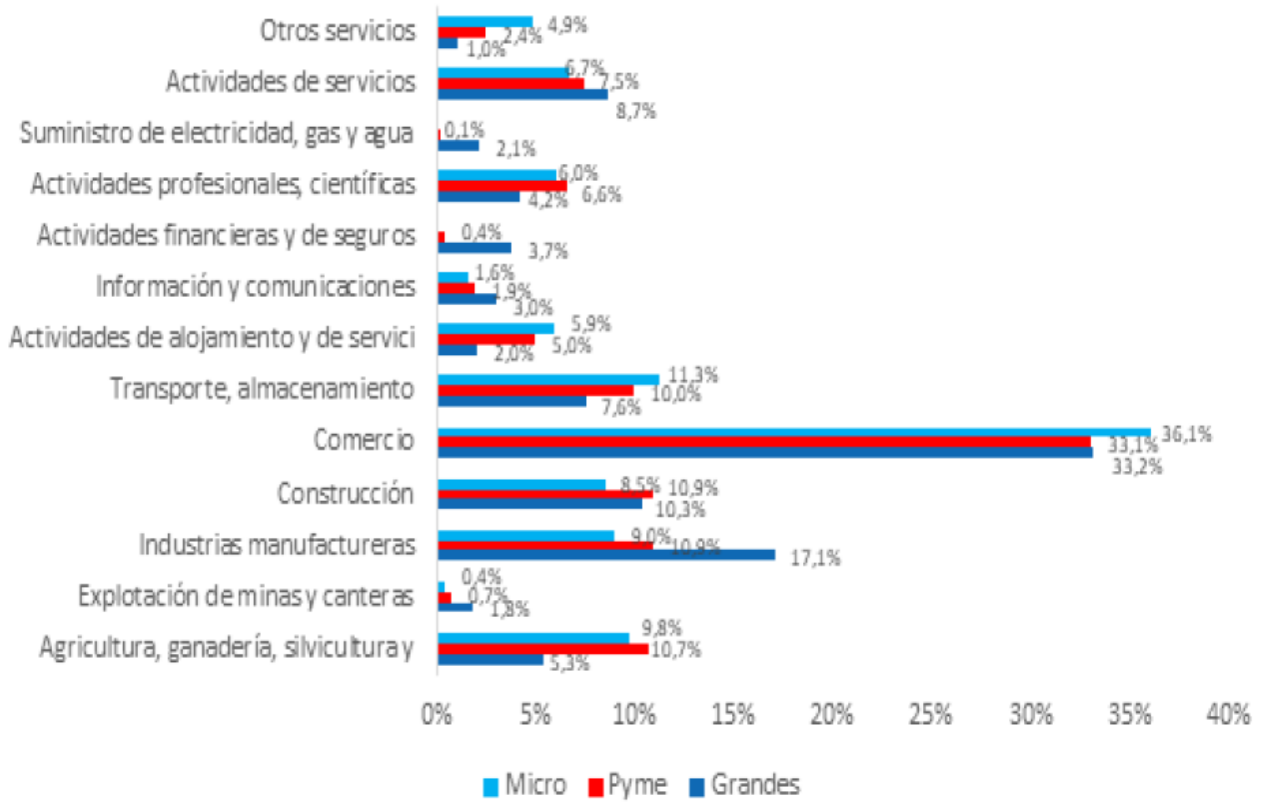

Grafico 1. Composición de empresas por sector y tamaño

Fuente: Cuarta Encuesta Longitudinal de Empresas, 2017

De estos sectores los que generan las principales exportaciones en las pymes son el de agrosilvicultura y pesca con un total de $18,9 \%$ exportado, seguido del sector de comunicaciones con un $11,6 \%$, adicional al movimiento generado por el sector del cobre. (Fuente Cuarta encuesta longitudinal de empresas unidad de estudios agosto 2017 pág. 4, y 27).

Las empresas que se encuentran en estos sectores deben competir con grandes empresas, como retail y grupos económicos del país en cuanto a nicho, precio, y calidad, adicional a restricciones del mercado financiero, por ello el posicionamiento y por ende el sostenimiento es una tendencia en las economías del mundo, ya que en todas se presentan este tipo de restricciones que hace que fracasen las empresas y no puedan concluir su ciclo.

De acuerdo con lo expuesto anteriormente es importante que el gobierno volqué su mirada a dichas empresas, con el fin de impulsar su crecimiento teniendo en cuenta los impactos contributivos que generan en cuanto a empleo y crecimiento económico sin desmeritar su aporte social. 


\subsection{Impacto Social}

En la actualidad, la Responsabilidad Social Empresarial es un aspecto vital en las empresas debido su contribución social y de medio ambiente, la RSE debe ser adoptada por todo tipo de empresas no importando su tamaño, y no le significaría incurrir en más costos para operar. Con la iniciativa que toma una empresa en este aspecto aportarían en materia no solo de beneficios para su gestión si no darían un gran aporte a la sociedad.

La RSE es un factor muy importante para el desarrollo económico de las pymes ya que es necesario para la competitividad y sustentabilidad en las cuales se integra por primera parte el respecto por los valores éticos, seguido del respecto a los colaboradores, a la comunidad y al medio ambiente.

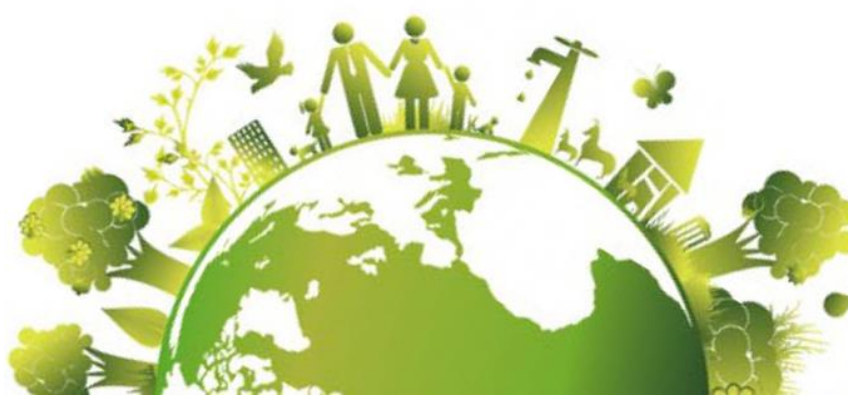

Figura 2. RSE

Fuente: Foto tomada de Internet

Resulta interesante ver como el RSE de las pymes en chile se genera a través de una serie de situaciones como son: responsabilidad por la sociedad, por valores y muchas otras por imagen. Las pequeñas y medianas empresas Chilenas tienen una importancia en la generación de empleo en el país, otorgan mucho trabajo a mujeres que se encuentran en regiones donde prima el desempleo, ellas han podido tener un crecimiento laboral y personal que ha desembocado en ideas de emprendimiento exitosas. Lo que ha generado un gran aporte a sus familias y estabilidad a su economía.

Dichas empresas también conciben compromisos con las comunidades locales en cuanto a la generación de actividades culturales, deportivas y de medio ambiente es por ello que estas buscan implementar nuevas formas de hacer las cosas y se resaltan como un gran modelo de contribución económica social.

\section{Instituciones que apoyan el emprendimiento chileno}

Chile es uno de los países más competitivos de Latinoamérica (GEM, 2017), lo anterior, debido a su eficiencia e innovación; así mismo, registran el mayor volumen de iniciativas emprendedoras generadas por la identificación de nuevas oportunidades, fomentando un crecimiento en los puestos de trabajo. 
Por esta razón, fueron creadas diversas instituciones para el desarrollo del emprendimiento en Chile, porque ayudan a fortalecer las pymes en el país, con herramientas que orientan a los emprendedores y empresarios. A continuación, se describen 6 instituciones que apoyan el emprendimiento:

- Centro de Desarrollo de Negocios de Santiago: con el lema "Tu proyecto + nuestra asesoría un mejor negocio", el Centro de Desarrollo de Negocios busca incentivar a las personas que quieran emprender una nueva idea de negocio. Es una institución que está en manos de Sercotec, la corporación de derecho privado dependiente del Ministerio de Economía, Fomento y Turismo.

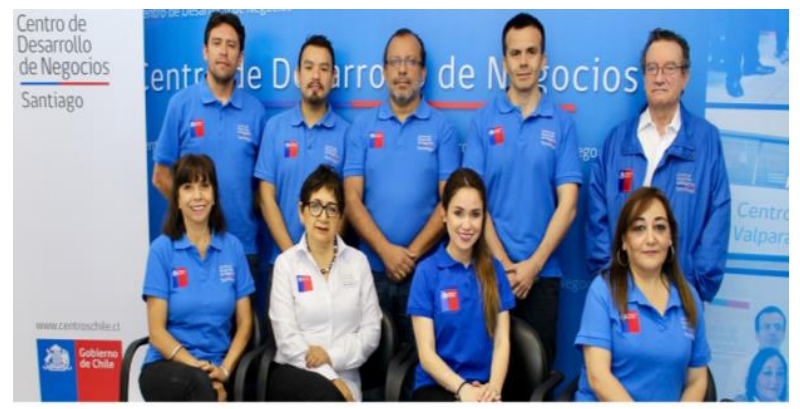

Figura 3. Equipo de asesores del CDN

Fuente: Centro de Desarrollo de Negocios, 2017

Nació en junio de 2014 como un acuerdo entre los gobiernos de Chile y Estados Unidos, por lo tanto, las pequeñas y medianas empresas cuentan con asesoría técnica para el crecimiento, la productividad y sostenibilidad de las mismas.

Así mismo, presta servicios para empresas establecidas con asesoría inicial, asesoría a largo plazo, finalmente teniendo como resultado el crecimiento de la empresa.

Adicionalmente, asesora empresas incipientes a través de un seminario llamado "cómo empezar su propio negocio", donde los instruyen para desarrollar un plan de negocio en borrador, terminando con el desarrollo final, luego el lanzamiento y finalmente el crecimiento de la empresa (Centros de Desarrollo de Negocios, 2018).

- ProChile: es una institución que ayuda a la internacionalización de las empresas, a través de la exportación, es decir, busca aumentar el rendimiento y el trabajo de las organizaciones públicas y privadas. Adicionalmente, brinda apoyo para tener variedad de productos y servicios generando oportunidades de negocio. Sus profesionales orientan a los exportadores, brindándoles información que les facilitará la internacionalización de las empresas, promocionando los productos a nivel mundial en Ferias Internacionales, Fondos Concursables y Marcas Sectoriales (Prochile, 2018).

Asimismo, implementa herramientas para potencializar competencias de gestión comercial tales como: "Exportar Paso a Paso", "Programa de Talleres" y "Programa Coaching". 


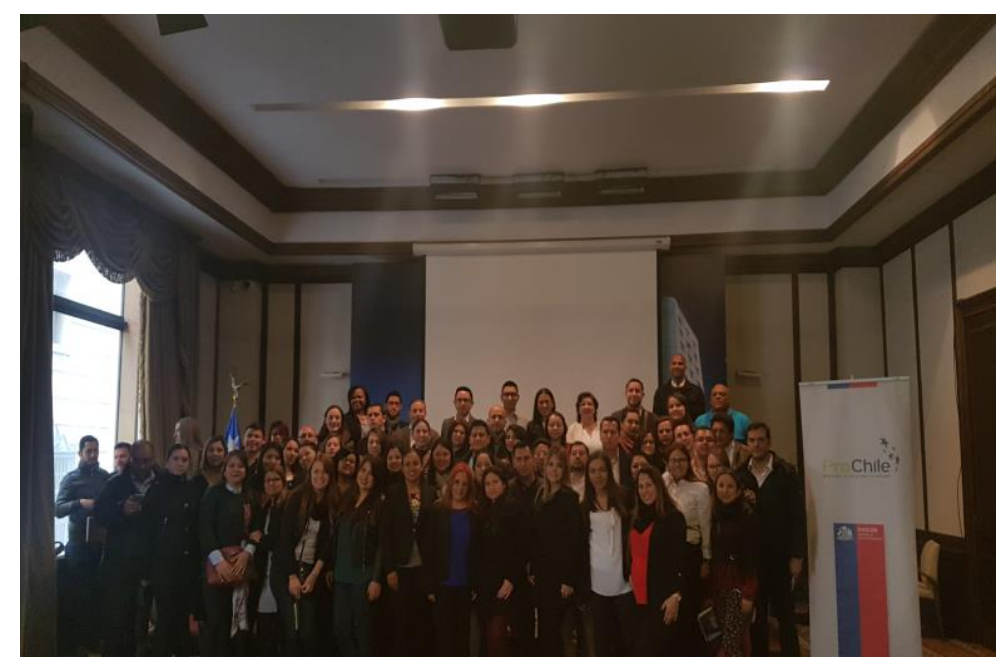

Figura 4. Instalaciones Prochile

Fuente: Los autores

- ASECH: con su lema "hacer de Chile el mejor país para emprender", esta asociación busca que todas las personas sin importar su origen, edad y el sector en el que decidan emprender, tengan una oportunidad para iniciar su proyecto. Su plan de motivación cuenta con cinco pilares que inspiran el valor de la valentía:

- Voz de los emprendedores. asesorías legales de forma preventiva y correctiva.

- Información. información útil, charlas online y avances del mercado a los que se tienen que enfrentar.

- Redes. generan redes de contacto, por lo tanto, realizan eventos masivos tal como ferias, seminarios.

- Formación. talleres que ayudan a potenciar el negocio generando estrategias para escalar sin dificultad.

- Defensa. asesorías legales.

- CEPAL: esta institución se fundó con el objetivo de contribuir a la economía de América Latina, a través de su estrategia de apoyo llamada Euromipyme, esta permite generar alianzas estratégicas buscando mejorar las condiciones productivas de las pequeñas y medianas empresas, así mismo, fortalecer la mano de obra, tener un producto innovador y hacer un estudio más detallado de los mercados, además hay seminarios y talleres etc. Este apoyo define una serie de herramientas orientadas a corregir las fallas de mercadeo, productividad y competitividad. Puesto que las Pymes representan una realidad productiva en Chile, con un $50 \%$ de los ocupados trabajan en este tipo de empresas (Cepal, 2018).

- Acción Emprendedora y Fundación independízate: estas 
dos instituciones se dedican a la formación y aprendizaje de los pequeños empresarios, a su vez, se enfocan en temas de gestión para el desempeño de las Pymes y no solo en el tema financiero (Acción emprendedora, 2018).

Tienen como objetivo, encaminar voluntariamente a estudiantes universitarios para desarrollar sus ideas de negocio, así mismo, les brindan cuatro herramientas para hacerlo posible: en primer lugar Arma tu Empresa, segundo jurídica, tercero Contable y por ultimo Marketing.

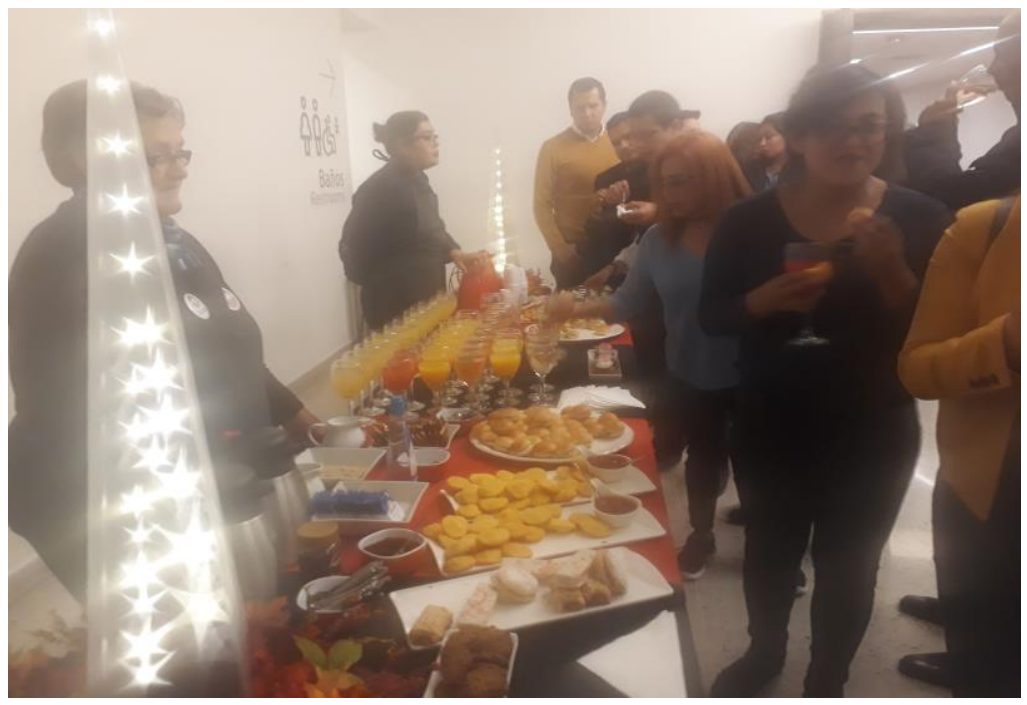

Figura 5. Muestra de Emprendimiento CDN Fuente: Los autores

- EmprendeFCh: esta plataforma de emprendimiento busca aumentar el desarrollo económico del país. Justamente apoya emprendimientos de alto potencial en 3 aspectos como lo son: tecnología, desarrollo del modelo de negocio y comercialización. Es considerada, como una alternativa aceleradora de negocios enfocados a la innovación (Fundación Chile).

\section{Alternativas de financiación con las que cuentan las Pymes en Chile}

En la actualidad, uno de los elementos más decisivos a la hora de crear empresa es el factor económico, pues no es suficiente tener una buena idea de negocio si no se cuenta con las herramientas para hacerlo posible. Según un análisis realizado por "ASECH" Asociación de emprendedores de Chile, la principal causa de fracaso de los emprendedores es la falta de financiación para el desarrollo de sus proyectos (Publímetro Emprende, 2016), en ocasiones debido a que los pequeños y medianos empresarios no estructuran de forma acertada el valor de su inversión o se les dificulta el acceso al crédito debido a las exigentes políticas del sector bancario. 
Teniendo en cuenta lo anterior, la decisión más importante que debe tomar un emprendedor es la forma como financiara su plan de negocio, por tanto, indagamos que alternativas prefieren en el país chileno.

Podemos decir, que la cultura del emprendedor chileno se prepara para sobrellevar los obstáculos que se presentan en el camino, es por esto, que el 58.1\% de los emprendedores optan por utilizar ahorros o recursos propios al momento de arrancar su negocio (INE, 2017), esto le genera ventajas tales como: disminución de trámites legales, ahorro al no tener que pagar intereses, evita riesgos empresariales por falta de solvencia, por consiguiente, autonomía para la toma de decisiones. Al contrario, el 16.1\% de los empresarios afirma utilizar préstamos bancarios como principal fuente de financiación, siendo conscientes que no solo hay que devolver el capital del préstamo, sino que la entidad financiera aplica una tasa de interés sobre ese capital, lo que radica como la principal desventaja de este tipo de financiación.

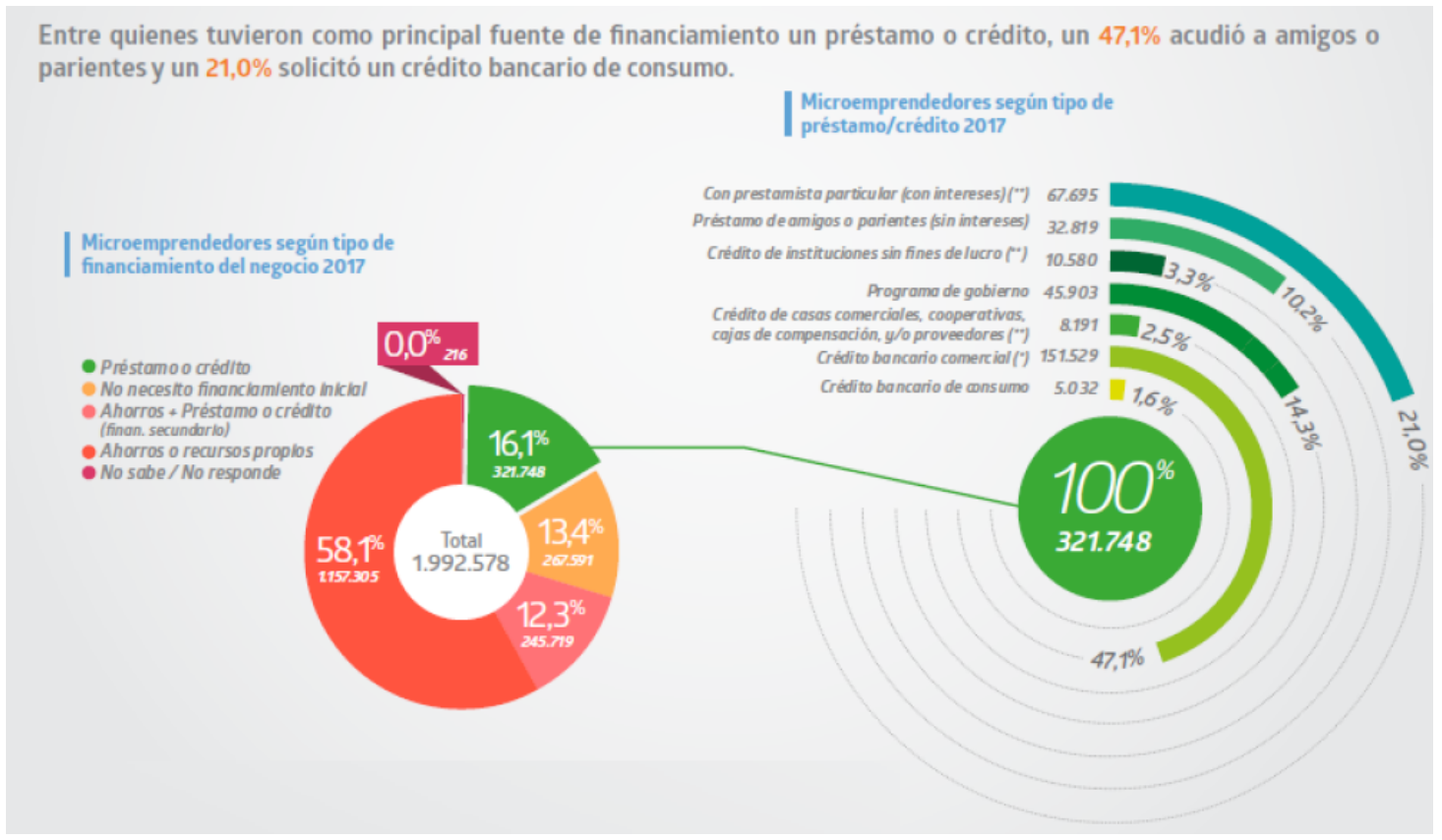

Grafico 2. Micro emprendedores según tipo de financiamiento Fuente: Encuesta de Micro emprendimiento, 2017

De otro lado, los emprendedores también cuentan con los fondos concursables (Broseta Andrea, 2018, Rankia), los cuales son instituciones que asignan recursos públicos y privados con el objetivo de financiar proyectos innovadores que permitan el desarrollo social y promover alianzas estratégicas entre los distintos sectores. Estos fondos buscan no solo apoyar a los emprendedores, sino a su vez generar una responsabilidad social con iniciativas como: mejorar la situación de pobreza y vulnerabilidad de las personas, mejorar sus condiciones de vivienda y lograr la inclusión social de mujeres, niños y jóvenes que infringen la ley. 
A continuación, describiremos brevemente los fondos concursables, donde los emprendedores pueden postularse para llevar a cabo sus proyectos:

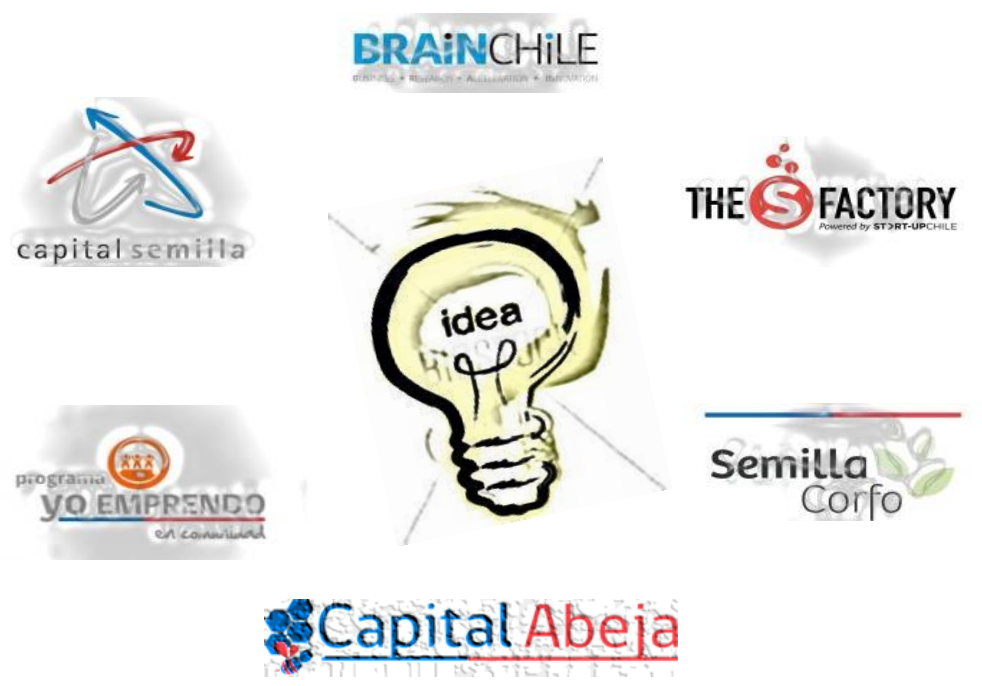

Figura 6. Fondos concursables

Fuente: Los autores

- Brain Chile: es otorgado por la Universidad Católica de Chile y el Banco Santander, con el objetivo de impulsar proyectos científicos-tecnológicos de instituciones de educación Superior Chilenas; este cuenta con un capital de 70 millones de pesos chilenos que se debe distribuir en los distintos proyectos seleccionados, con la condición de proporcionar espacios de trabajo, laboratorios, mentores y entrenamiento en emprendimiento.

- Capital Semilla: este programa asigna un subsidio de 3,5 millones de pesos chilenos no reembolsables, con el fin de afianzar los proyectos en áreas de asistencia técnica, Marketing, acompañamiento, capacitaciones e inversiones, es otorgado por Sercotec, una corporación que depende del Ministerio de Economía, fomento y turismo dedicada al apoyo de las micro y pequeñas empresas.

- Programa yo emprendo: este fondo concursable aplica paras las personas que ya cuentan con un negocio en marcha, ofreciéndole talleres de emprendimiento, visitas de asesoría y financiamiento de un plan de negocios, de manera que el empresario pueda hacer crecer su negocio y aumentar sus ganancias.

- Thes Factory: otorga 10 millones pesos chilenos por medio de la agencia de gobierno chileno Corfo, con la intención de buscar mujeres innovadoras que transformen sus ideas en modelos funcionales, a través de entrenamiento para los emprendedores y mentores especializados. 
- Capital Abeja: también otorgado por Sercotec para apoyar el comienzo de nuevos negocios liderados por mujeres que puedan participar en el mercado. El monto aplica de acuerdo a su destinación y pueden ser utilizados en asistencia técnica, capacitación, marketing, acompañamiento e inversiones con el fin de afirmar el proyecto.

- Semilla Corfo: este se asigna para proyectos con una duración máxima de 12 meses que puedan alcanzar un monto de ventas de 1 millón de pesos chilenos al acabo de 3 años, y doblar sus ingresos cada 3 años.

Por último, podemos concluir que los pequeños y medianos empresarios deben buscar la alternativa de financiación más acertada para su negocio, con el fin de lograr un equilibrio económico que permita su apalancamiento y duración en el mercado; lo anterior, se logra con una buena planeación del modelo de negocio, que incluya el valor de la inversión, la innovación y creatividad.

\section{El futuro de las Pymes}

Como hemos mencionado, el mercado chileno busca emprendedores que vayan de la mano con las necesidades y expectativas del consumidor, ya que hoy en día en un mundo tan globalizado la competencia es cada vez más fuerte y exigente. Por esta razón, vale la pena resaltar el papel que cumple la innovación en el desarrollo del mundo empresarial, pues no solo obtiene mayores beneficios económicos, sociales y tecnológicos, sino también asegura su durabilidad a lo largo del tiempo.

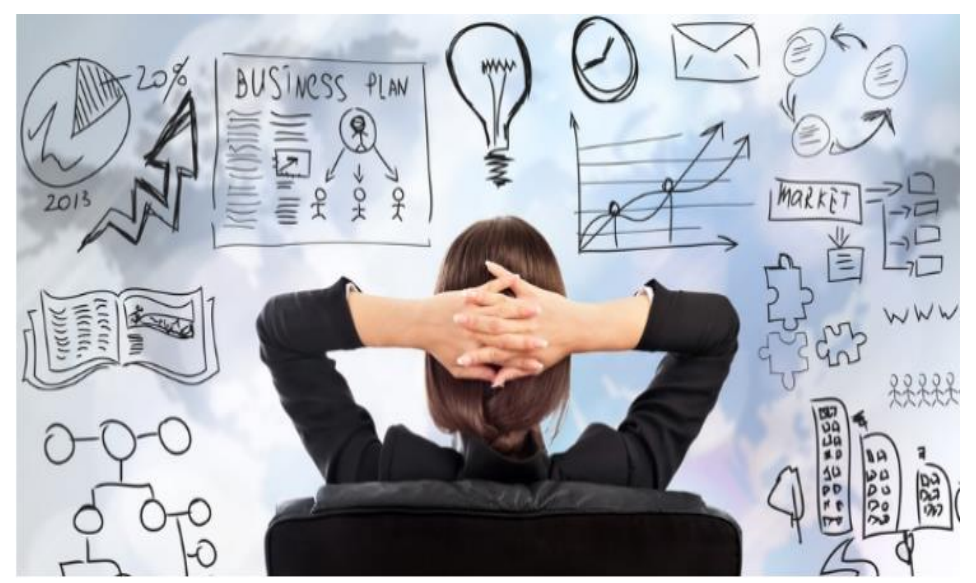

Figura 7. Emprendimiento

Fuente: Centro de Desarrollo de Negocios, 2018 
La innovación es una excelente herramienta para aumentar la productividad de las pequeñas y medianas empresas e impulsarlas para lograr su crecimiento; por lo anterior, hablaremos de un tema que está de moda y se ha vuelto imprescindible para las organizaciones.

La tecnología sirve como medio para implementar cambios de manera rápida, así mismo ayuda a transformar ágilmente las ofertas y servicios de acuerdo a las necesidades del cliente. Es por esto, que vale la pena mencionar la incursión que las Pymes han tenido en los canales electrónicos; y es que según estudios de la Cámara de Comercio de Santiago (Cámara de comercio de Santiago, 2018), las ventas en línea durante el año 2017 superaron los 3.700 millones de dólares y se espera que para el 2020 la cifra alcance los 8.000 millones de dólares. Así mismo, ocurre con el programa de "Pymes Digitales" lanzado por el gobierno chileno, esta plataforma le permite a los emprendedores y pymes reducir muchos trámites relacionados con su desarrollo, mejorar el acceso a la conectividad y crear sistemas de capacitación en línea.

Ahora bien, las cifras anteriores demuestran como las pequeñas y medianas empresas están creciendo e incrementado de manera significativa sus ingresos, demostrando de esta manera la importancia en la economía chilena. Por lo anterior, se hace indispensable mencionar que las proyecciones para el año 2018 apuntan que las Pymes tendrán nuevamente un aumento en sus ventas y que a su vez contribuirán con el desarrollo económico del país.

De otro lado, conforme a los pronósticos del Banco Central de Chile, el país tendrá un crecimiento en su producto interno bruto PIB que se situara para el mes de diciembre en 3.5\%, esto lo proyecta basado en la recuperación económica que ha tenido el país y que se refleja con las cifras de meses como febrero de 2018 donde su crecimiento fue del 3.3\% en comparación con meses anteriores (Diario El Centro, Marzo 2018).

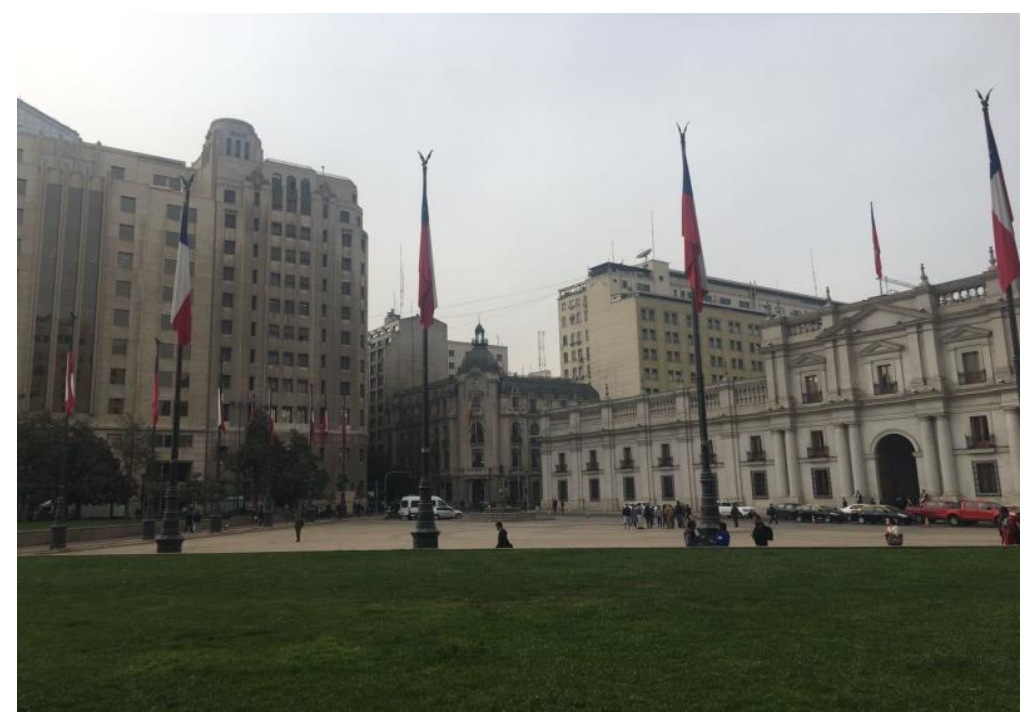

Figura 8. Plaza de la Constitución, Santiago de Chile

Fuente: Los autores 
Ahora bien, las Pymes tienen una relación directa con los ciclos económicos y su tasa de creación va ligada con el crecimiento del PIB, por tanto, estas proyecciones mantienen una gran expectativa en este sector (El Heraldo, 2018).

Para finalizar, podemos concluir que este cambio económico y la penetración en nuevas herramientas tecnológicas lleva a pensar que los micro y pequeños empresarios tendrán un mejor futuro, con un mayor crecimiento y un incremento significativo en sus ventas.

\section{Conclusiones}

La pequeña y mediana empresa en Chile representa, un aporte importante a nivel económico y social, su contribución en la generación de empleos y producción nacional ha permitido que dicho país se consolide como uno de los principales a nivel Latinoamérica en la implementación de proyectos de emprendimiento e innovación lo que muestra que una idea de negocio se puede convertir en una gran empresa.

Sin duda las pymes hacen parte del desarrollo histórico del patrimonio de chile, en términos internacionales, las exportaciones de productos chilenos han logrado superar la de otros países, por lo tanto, Chile es segunda economía de ingresos dependientes de exportaciones.

Podemos afirmar que, para conseguir la durabilidad de las pequeñas y medianas empresas, los emprendedores deben escoger la alternativa de financiación que le permita mantener un equilibrio económico, así mismo realizar una buena planeación del modelo de negocio garantizando que podrá reaccionar ante situaciones inesperadas.

El futuro de chile está en transformar, innovar y motivar a las personas para ir más allá y crear un bienestar a la sociedad y el país; es por eso que cuentan con distintas instituciones que dan apoyo a las pymes, lo que buscan es descubrir proyectos que cambien la realidad económica del país y que generen oportunidades de empleo. Estas instituciones promueven este emprendimiento desde la educación teniendo como resultado la generación de nuevas ideas de negocio que ayuden al progreso del país.

Las Pymes deben estar a la vanguardia del mundo actual, incursionando con herramientas tecnológicas que permitan optimizar sus procesos para que sean más competitivos y a su vez se refleje en sus resultados financieros.

La restricción en el financiamiento de la pequeña y mediana empresa en cuanto al sector bancario se observa a nivel mundial lo que ha generado que muchas de estas empresas no puedan concluir su ciclo contando con ideas exitosas, dicho factor genera un impacto negativo para su crecimiento. Con relación a esta restricción Seria importante que el sector financiero evaluara sus políticas con el fin de generar una mayor contribución.

La responsabilidad social empresarial puede considerarse como una herramienta estratégica que influye de manera directa en la competitividad de la pyme, ya que genera unos beneficios tanto económicos como sociales, que se convierten en una mayor sostenibilidad de la empresa. 
Así mismo, al ser responsables socialmente se incrementa la productividad y esto su vez mejora las condiciones de los trabajadores y de la sociedad de un país.

De acuerdo a la experiencia obtenida en la Misión académica, se pudo observar, que Chile presenta un crecimiento económico, diferencia cultural y emprendimiento a través de las visitas realizadas, cabe destacar que es un país con un gran aporte en ideas de innovación promovidas por organizaciones que buscan que estas concluyan con gran éxito. 


\section{Referencias Bibliográficas}

[1] Diario El Centro. Noticias Económicas. [en línea], Expertos suben al 3,5\% proyección de crecimiento en 2018 para Chile, 2018 [consulta, 22/31 de mayo de 2018]. Disponible en: http://www.diarioelcentro.cl/noticias/economia/expertos-suben-al-35-proyeccion-decrecimiento-en-2018para-chile

[2] Fajardo Daniel. El pulso. Empresas y Mercados. [en línea], El 72\% de las pymes cree que la economía mejorará en el primer semestre de 2018, 2018 [consulta, 22/31 de mayo de 2018]. Disponible en: http://www.pulso.cl/empresas-mercados/72-las-pymes-cree-la-economiamejorara-primersemestre-2018/

[3] Cesla. Noticias relacionadas con economía. [en línea], Chile ve recuperación generalizada y sostenida de su economía, 2018 [consulta, 22/31 de mayo de 2018]. Disponible en: https://www.cesla.com/detalle-noticias-de-chile.php?Id=2396

[4] Comunidad Empresas. Artículos. [en línea], La realidad de los microemprendedores en Chile, 2018 [consulta, 22/31 de mayo de 2018]. Disponible en: https://ce.entel.cl/articulos/realidad-microemprendedores-en-chile/

[5] Diario El Heraldo. Noticias. [en línea], ¿Cuáles son las proyecciones para las Pymes este 2018?, 2018 [consulta, 22/31 de mayo de 2018]. Disponible en: http://www.diarioelheraldo.cl/noticia/cuales-son-las-proyecciones-para-las-pymes-este-2018

[6] Ángel Cabrera, Sergio de la Cuadra, Alexander Galetovic y Ricardo Sanhueza. Cepchile. [en línea], LAS PYME: Quiénes Son, Cómo Son Y Qué Hacer Con Ellas, 2018 [consulta, 22/31 de mayo de 2018]. Disponible en: https://www.cepchile.cl/cep/site/artic/20160304/asocfile/20160304095131/rev116_AGaletov ic_otros.pdf

[7] Comunidad Empresas. Artículos. [en línea], Chile son mi Pymes, 2018 [consulta, 22/31 de mayo de 2018]. Disponible en: https://ce.entel.cl/articulos/96-7-empresas-chile-son-mipymes/

[8] Cepal. Noticias. [en línea], Estrategias de apoyo a las mipymes de la región, 2018 [consulta, 22/31 de mayo de 2018]. Disponible en: https://www.cepal.org/es/search?as_q=mipymes\%20en\%20chile

[9] Fajardo Daniel. El pulso. Empresas y Mercados. [en línea], Nuevo estudio AVLA-Cadem: pymes cada vez más optimistas acerca del futuro económico, 2018 [consulta, 22/31 de mayo de 2018]. Disponible en: http://www.pulso.cl/empresas-mercados/nuevo-estudio-avla-cadempymes-vez-mas-optimistas-acerca-del-futuro-economico/

[10] Ministerio de Economía, Fomento y Turismo. Empresas, pymes y emprendimiento. [en línea], Quinta Encuesta de Microemprendimiento (EME5), 2018 [consulta, 22/31 de mayo de 
2018]. Disponible en: http://www.economia.gob.cl/category/estudiosencuestas/emprendimiento.

[11] Oyarce Gerty. Publimetro. Emprendimientos. [en línea], Cuatro emprendimientos hechos por chilenas que tienes que conocer, 2018 [consulta, 22/31 de mayo de 2018]. Disponible en: https://www.publimetro.cl/cl/noticias/2018/03/08/emprendimientos-chilenas.html

[12] Qué es una Pyme | Importancia de las Pymes en Chile 11 de marzo 2017 Disponible en: https://opcionis.cl/blog/pymes-en-chile/

[13] Rodrigo Krell, Eduardo Jiménez, Miguel Jara, Sandra Peralta, Tomás Schuster. Informe de resultados empresas en chile. MINISTERIO DE ECONOMÍA OMENTO Y TURISMO, Agosto 2017. División de Política Comercial e Industrial. Boletín-empresas-en-Chile-ELE4.

[14] Milano Néstor. Director Ejecutivo de Laborum Chile, Perú \& Venezuela. La Importancia de las PYMES en la generación de trabajo en América Latina, 2016. Disponible en: http://pyme.emol.com/3632/importancia-pymes-en-el-trabajo-latam/

[15] Servicio de impuestos interno. Estadísticas de empresas por tamaño según ventas, 2016. Disponible en: http://www.sii.cl/estadisticas/empresas_tamano_ventas.htm

[16] Capacitarse. Cursos. [en línea], El estado de la RSE en las pymes de chile, 2018 [consulta, 09 de Junio de 2018]. Disponible en: http://www.cursosderse.com/2011/03/estudioresponsabilidad-social-pymes-chile/

[17] Revista Emprende. [en línea], Las Pymes son un motor fundamental en lo económico y en lo social, 2018 [consulta, 22/31 de mayo de 2018]. Disponible en: http://www.revistaemprende.cl/las-pymes-motor-fundamental-lo-economico-lo-social/

[18] Publimetro. [en línea], ¿Cómo financiar un emprendimiento? Conoce las alternativas, 2016 [consulta, 22/31 de mayo de 2018]. Disponible en: https://www.publimetro.cl/cl/diariopyme/2016/07/08/financiar-emprendimiento-conoce-alternativas.html

[19] Emol.com. Noticias Económia. [en línea], Ocho de cada 10 emprendimientos fracasan en Chile: Experto entrega claves para evitar errores, 2018 [consulta, 22/31 de mayo de 2018]. Disponible en: http://www.emol.com/noticias/Economia/2018/04/22/903519/Asesor-deemprendedores-Hoy-toda-venta-tiene-que-centrarse-en-hacerle-la-vida-mas-facil-alcliente.html

[20] Agenda emprendedora. Noticias. [en línea], Emprendimiento e Innovación de clase mundial "Made-in-Chile", 2018 [consulta, 22/31 de mayo de 2018]. Disponible en: http://www.elmostrador.cl/agenda-pais/2018/03/22/emprendimiento-e-innovacion-de-clasemundial-made-in-chile/ 
[21] Instituto Nacional de Estadisticas. [en línea], Informe final, 2018 [consulta, 22/31 de mayo de 2018]. Disponible en: http://www.economia.gob.cl/2018/02/13/quinta-encuesta-demicroemprendimiento-eme5.htm

[22] Universia. [en línea], 7 fondos concursables para financiar tu emprendimiento en Chile, 2017 [consulta, 22/31 de mayo de 2018]. Disponible en: http://noticias.universia.cl/practicasempleo/noticia/2016/04/12/1138178/7-fondos-concursables-financiar-emprendimientochile.html

[23] Instituto Nacional de Estadisticas.. [en línea], Cuarta Encuesta Longitudinal de Empresas, 2017 [consulta, 22/31 de mayo de 2018]. Disponible en: http://www.economia.gob.cl/estudiosy-encuestas/cuarta-encuesta-longitudinal-de-empresas-ele-4

[24] Vernon Romina. Networker Chile. [en línea], Pymes en Chile, el sector más endeudado del 2017, 2017 [consulta, 22/31 de mayo de 2018]. Disponible en: https://networker.cl/pymeschile-sector-mas-endeudado-del-2017/

[25] Castillo Camila. El pulso. Empresas y Mercados. [en línea], Radiografía a las pequeñas y medianas empresas chilenas, 2017 [consulta, 22/31 de mayo de 2018]. Disponible en: http://www.pulso.cl/empresas-mercados/radiografia-las-pequenas-medianas-empresaschilenas/

[26] Fondos concursables. [en línea], FONDOS CONCURSABLES A LOS QUE PUEDE POSTULAR, 2018 [consulta, 22/31 de mayo de 2018]. Disponible en: http://www.doh.gov.clAPRdocumentosDocumentsfondos_foncursables.pdf

[27] Andrea Boseta. Rankia Chile. [en línea], Financiamiento para emprendedores Chile 2018, 2018 [consulta, 22/31 de mayo de 2018]. Disponible en: https://www.rankia.cl/blog/ideasemprendimiento-chile/3585260-financiamiento-para-emprendedores-chile-2018 